\title{
Fruit and vegetable intakes and the association with blood pressure within adults in the United Kingdom's National Diet \& Nutrition Survey Rolling Programme (2008/09-2011/12)
}

\author{
H.T. Lai, M. Holmes, G. Williamson and V.J. Burley \\ School of Food Science \& Nutrition, University of Leeds, Leeds LS2 9JT, UK
}

This abstract was awarded the student prize for best poster original communication.

In intervention studies, increased fruit and vegetable $(\mathrm{F} \& \mathrm{~V})$ intake is associated with a decreased risk of hypertension ${ }^{(1-2)}$. Observational studies also report consistent inverse associations ${ }^{(3)}$. However, fruit or vegetable type has been less frequently explored. This study aimed to explore the association between total fruit and vegetable intakes, type of fruit and vegetables according to polyphenol content and blood pressure $(\mathrm{mmHg})$ in the National Diet and Nutrition Survey Rolling Programme from 2008 to 2012.

Total fruit and total vegetable intakes (g/day) derived from 3-4 day food diaries obtained from 1,002 adults (age range 19 to 91 years) after exclusions were explored. Fruit and vegetable intakes were sub-categorised according to similarities in polyphenol content from Phenol Explorer ${ }^{(4)}$, including berries, citrus, drupes, pomes and tropical fruits for fruits; Allium, Brassicaceae, fruit vegetables, pod vegetables and root vegetables for vegetables. Valid blood pressure $(\mathrm{mmHg})$ results were used as the measurement outcome. Multivariate regression was conducted to calculate change in systolic (SBP) and diastolic blood pressure (DBP) (mmHg) per unit increase in F\&V in adults (19+ years) not taking blood pressure related medication. Change in blood pressure was determined by comparing each intake group with the reference group (data not shown). Linear trend was also determined by $80 \mathrm{~g}$ portions of fruit and vegetables.

Dietary intakes and $\beta$-coefficient for change in systolic and diastolic blood pressure $(\mathrm{mmHg})$

\begin{tabular}{|c|c|c|c|c|}
\hline \multirow[b]{2}{*}{ Exposure ( $80 \mathrm{~g} /$ day) } & \multicolumn{2}{|c|}{ SBP $(\mathrm{mmHg})\left(95 \%^{\mathrm{a}}\right.$ or $\left.99 \%{ }^{\mathrm{b}} \mathrm{CI}\right)$} & \multicolumn{2}{|c|}{ DBP $(\mathrm{mmHg})\left(95 \%{ }^{\mathrm{a}}\right.$ or $\left.99 \%{ }^{\mathrm{b}} \mathrm{CI}\right)$} \\
\hline & Age Adjusted & Fully Adjusted & Age Adjusted & Fully Adjusted \\
\hline Total Fruits \& Vegetables ${ }^{a}$ & $-0.57(-0.98,-0.15)^{* *}$ & $-0 \cdot 58(-1 \cdot 00,-0 \cdot 15)^{* *}$ & $-0.45(-0.75,-0.15)^{* *}$ & $-0.43(-0.74,-0.12)^{* *}$ \\
\hline Total Fruits $^{\mathrm{a}}$ & $-0.91(-1 \cdot 60,-0 \cdot 22)^{* *}$ & $-0.65(-1.37,0.07)$ & $-0.95(-1.44,-0.45)^{* *}$ & $-0.82(-1.34,-0.29)^{* *}$ \\
\hline Berries ${ }^{b}$ & $-1.49(-4 \cdot 32,1 \cdot 34)$ & $-0.83(-3.64,1.97)$ & $-1 \cdot 17(-3 \cdot 23,0 \cdot 88)$ & $-1.02(-3.04,0.99)$ \\
\hline Citrus ${ }^{b}$ & $0.10(-0.81,1.02)$ & $-0.47(-1.68,0.74)$ & $-0.30(-0.97,0 \cdot 36)$ & $-0.96(-1.83,-0.09)^{* *}$ \\
\hline Drupes ${ }^{b}$ & $2 \cdot 64(-1.09,6.38)$ & $3.54(-0 \cdot 10,7 \cdot 18)$ & $-0.75(-3.46,1.96)$ & $0.09(-2.56,2.74)$ \\
\hline Pomes ${ }^{b}$ & $-1.18(-2.75,0.38)$ & $-1.00(-2.57,0.56)$ & $-1.29(-2.43,-0.16)^{* *}$ & $-1 \cdot 14(-2 \cdot 27,-0 \cdot 01)^{* *}$ \\
\hline Tropical Fruits ${ }^{b}$ & $-0.62(-2.93,1.69)$ & $0.33(-2 \cdot 04,2 \cdot 70)$ & $-0.93(-2 \cdot 60,0.75)$ & $-0.09(-1.81,1.63)$ \\
\hline Total Vegetables ${ }^{a}$ & $-0.60(-1.25,0.05)$ & $-0 \cdot 52(-1 \cdot 20,0 \cdot 16)$ & $-0.28(-0.76,0.19)$ & $-0.07(-0.57,0.42)$ \\
\hline Allium ${ }^{b}$ & $-0.92(-5 \cdot 21,3 \cdot 36)$ & $-0 \cdot 31(-4 \cdot 78,4 \cdot 16)$ & $0 \cdot 31(-2 \cdot 80,3 \cdot 42)$ & $0.64(-2 \cdot 60,3 \cdot 87)$ \\
\hline Brassicaceae $^{b}$ & $-2.83(-6.53,0.87)$ & $-1.48(-5.24,2.27)$ & $-1.62(-4.31,1.06)$ & $-0.62(-3 \cdot 34,2 \cdot 09)$ \\
\hline Fruit Vegetables ${ }^{\mathrm{b}}$ & $-0.89(-2.80,1.02)$ & $0.13(-1.89,2 \cdot 16)$ & $-0.44(-1.83,0.94)$ & $0.27(-1.20,1.73)$ \\
\hline Pod Vegetables ${ }^{b}$ & $-0.68(-5.85,4.48)$ & $-0.42(-5.41,4.56)$ & $0.28(-3.47,4.02)$ & $0.88(-2.72,4.49)$ \\
\hline Root Vegetables ${ }^{b}$ & $-1.83(-4.43,0.77)$ & $-0 \cdot 50(-3 \cdot 20,2 \cdot 19)$ & $-1.06(-2.95,0.82)$ & $-0 \cdot 12(-2 \cdot 07,1 \cdot 82)$ \\
\hline
\end{tabular}

If $95 \%{ }^{\text {a }} \mathrm{CI}$, significance level is $* P<0.05$; if $99 \%{ }^{\mathrm{b}} \mathrm{CI}$, significance level is $* * P<0.01$

Systolic and diastolic blood pressure was $-0.58 \mathrm{mmHg}(95 \% \mathrm{CI}-1 \cdot 00,-0 \cdot 15)$ and $-0.43(95 \% \mathrm{CI}-0.74,-0 \cdot 12) \mathrm{mmHg}$ lower respectively for each $80 \mathrm{~g} /$ day total F\&V consumed. Diastolic blood pressure was $-0.82 \mathrm{mmHg}(95 \% \mathrm{CI}-1 \cdot 34,-0 \cdot 29),-0 \cdot 96(99 \%$ $\mathrm{CI}-1.83,-0.09) \mathrm{mmHg}$ and $-1.14(99 \% \mathrm{CI}-2.27,-0.01) \mathrm{mmHg}$ lower respectively for $80 \mathrm{~g} /$ day intake of total fruit, citrus and pomes. Consumption of total fruits, citrus and pomes in particular was associated with a lower diastolic blood pressure in this cohort. Vegetable consumption was however, not associated with lower SBP or DBP measurements. These findings support guidelines encouraging fruit consumption for health, and provide evidence to suggest that higher intakes of citrus and pomes may be particularly beneficial in terms of lowering blood pressure, offering potential as a primary prevention to lower cardiovascular disease ${ }^{(5)}$.

1. Appel et al. (1997). New Engl J Med 336(16) 1117-1124

2. John et al. (2002). Lancet 359(9322) 1969-1974

3. Cassidy et al. (2011). Am J Clin Nutr 93(2) 338-347

4. Neveu et al. (2010). Database bap024 doi: 10.1093/database/bap024

5. Cook et al. (1995). Arch Intern Med 155(7) 701-709 